\title{
Canonical Structure of A and B Maps
}

\author{
Sudha ${ }^{1}$, B. N. Karthik ${ }^{2,3}$, A. R. Usha Devi ${ }^{2,3}$ \& A. K. Rajagopal ${ }^{3}$ \\ ${ }^{1}$ Department of Physics, Kuvempu University, Shankaraghatta, Shimoga, India. E-mail: tthdrs@gmail.com \\ ${ }^{2}$ Department of Physics, Bangalore University, Bangalore, India. \\ ${ }^{3}$ Inspire Institute Inc., Alexandria, Virginia, USA.
}

Editors: Vinayak Jagadish \& Danko Georgiev

Article history: Submitted on September 10, 2021; Accepted on October 22, 2021; Published on November 3, 2021.

n their seminal 1961 paper, Sudarshan, Mathews and Rau investigated properties of the dynamical $A$ and $B$ maps acting on $n$ dimensional quantum systems. The nature of dynamical maps in open quantum system evolutions has attracted great deal of attention in the later years. However, the novel paper on the $A$ and $B$ dynamical maps has not received its due attention. In this tutorial article, we review the properties of $A$ and $B$ forms associated with the dynamics of finite dimensional quantum systems. In particular, we investigate a canonical structure associated with the $A$ form and establish its equivalence with the associated $B$ form. We show that the canonical structure of the $A$ form captures the completely positive (not completely positive) nature of the dynamics in a succinct manner. This feature is illustrated through physical examples of qubit channels.

Quanta 2021; 10: 34-41.

\section{Introduction}

The conceptual formulation of dynamical $A$ and $B$ forms was pioneered by Sudarshan and coworkers 60 years ago [1,2]. The $A$ and $B$ matrices play an important role to identify if the open system dynamics of finite dimensional

(c) (1) This is an open access article distributed under the terms of the Creative Commons Attribution License CC-BY-3.0, which permits unrestricted use, distribution, and reproduction in any medium, provided the original author and source are credited. quantum systems is completely positive or not [3-6]. In this article, we study the dynamical $A$ and $B$ forms in detail. In particular, we investigate the canonical structure of the $A$ form and its properties. We show that there is a one-to-one connection between the canonical $A$ form and the $B$ form. We also construct the canonical $A$ form associated with some important physical examples of qubit channels [3].

In Section 2, we introduce the $A$ and $B$ forms and discuss their properties [1,2]. We present the canonical structure of the $A$ form and establish its equivalence with the $B$ form in Section 3, The canonical structure of the $A$ form is explicitly constructed for several qubit channels in Section 4 . Concluding remarks are given in Section 5 .

\section{Properties of A and B maps}

Consider a $n$ dimensional Hilbert Space $\mathcal{H}_{n}$. State of a quantum system is described by a density matrix $\rho \in \mathcal{H}_{n}$, defining properties of which are given by

1. Hermiticity: $\rho^{\dagger}=\rho$.

2. Unit trace: $\operatorname{Tr} \rho=1$.

3. Positivity: $\langle\psi|\rho| \psi\rangle \geq 0$ for all $|\psi\rangle \in \mathcal{H}$.

For a qubit (two-level quantum system) we have

$$
\rho=\frac{1}{2}(I+\vec{\sigma} \cdot \vec{p})
$$


where $I$ denotes $2 \times 2$ identity matrix, $\vec{\sigma}=\left(\sigma_{1}, \sigma_{2}, \sigma_{3}\right)$ by $\quad \vec{p}=\left(p_{1}, p_{2}, p_{3}\right) \quad$ satisfy $\quad$ the $\quad$ condition are Pauli matrices:

$|\vec{p}|=\sqrt{p_{1}^{2}+p_{2}^{2}+p_{3}^{2}} \leq 1$. Thus, the state space of $\sigma_{1}=\left(\begin{array}{cc}0 & 1 \\ 1 & 0\end{array}\right), \quad \sigma_{2}=\left(\begin{array}{cc}0 & -i \\ i & 0\end{array}\right), \sigma_{3}=\left(\begin{array}{cc}1 & 0 \\ 0 & -1\end{array}\right)$, a qubit corresponds to a unit ball in $\mathbb{R}^{3}$. The vector $\vec{p}$ is called the Bloch vector.

(2) Now we consider a linear map $A$ transand the real qubit state parameters given forming density matrices in $\mathcal{H}_{n}$ :

$$
\begin{aligned}
A: \quad \rho_{i} \rightarrow \rho_{f}=A\left(\rho_{i}\right), \\
\left(\rho_{f}\right)_{r^{\prime} s^{\prime}}=\left(A \rho_{i}\right)_{r^{\prime} s^{\prime}}=\sum_{r, s=1}^{n} A_{r^{\prime} s^{\prime} ; r s}\left(\rho_{i}\right)_{r s}, \quad r^{\prime}, s^{\prime}=1,2, \ldots, n .
\end{aligned}
$$

- The $n^{2} \times n^{2}$ matrix $A$ is called a trace-preserving positive map if, for every input density matrix, the output $\rho_{f}=A\left(\rho_{i}\right)$ is also a legitimate density matrix [1,2].

Writing the qubit density matrix (1) explicitly in the standard basis $|0\rangle=(1,0)^{T},|1\rangle=(0,1)^{T}$ as

$$
\rho=\left(\begin{array}{cc}
\rho_{00} & \rho_{01} \\
\rho_{10} & \rho_{11}
\end{array}\right)=\frac{1}{2}\left(\begin{array}{cc}
1+p_{3} & p_{1}-i p_{2} \\
p_{1}+i p_{2} & 1-p_{3}
\end{array}\right)
$$

one may identify the action $A: \rho_{i} \rightarrow \rho_{f}=A\left(\rho_{i}\right)$ (see (3)) as follows:

$$
\begin{aligned}
\left(\begin{array}{c}
\left(\rho_{f}\right)_{00} \\
\left(\rho_{f}\right)_{01} \\
\left(\rho_{f}\right)_{10} \\
\left(\rho_{f}\right)_{11}
\end{array}\right) & =\left(\begin{array}{llll}
A_{00 ; 00} & A_{00 ; 01} & A_{00 ; 10} & A_{00 ; 11} \\
A_{01 ; 00} & A_{01 ; 01} & A_{01 ; 10} & A_{01 ; 11} \\
A_{10 ; 00} & A_{10 ; 01} & A_{10 ; 10} & A_{10 ; 11} \\
A_{11 ; 00} & A_{11 ; 01} & A_{11 ; 10} & A_{11 ; 11}
\end{array}\right)\left(\begin{array}{c}
\left(\rho_{i}\right)_{00} \\
\left(\rho_{i}\right)_{01} \\
\left(\rho_{i}\right)_{10} \\
\left(\rho_{i}\right)_{11}
\end{array}\right) \\
\text { or }\left(\begin{array}{c}
1+\left(p_{f}\right)_{3} \\
\left(p_{f}\right)_{1}-i\left(p_{f}\right)_{2} \\
\left(p_{f}\right)_{1}+i\left(p_{f}\right)_{2} \\
1-\left(p_{f}\right)_{3}
\end{array}\right) & =\left(\begin{array}{llll}
A_{00 ; 00} & A_{00 ; 01} & A_{00 ; 10} & A_{00 ; 11} \\
A_{01 ; 00} & A_{01 ; 01} & A_{01 ; 10} & A_{01 ; 11} \\
A_{10 ; 00} & A_{10 ; 01} & A_{10 ; 10} & A_{10 ; 11} \\
A_{11 ; 00} & A_{11 ; 01} & A_{11 ; 10} & A_{11 ; 11}
\end{array}\right)\left(\begin{array}{c}
1+\left(p_{i}\right)_{3} \\
\left(p_{i}\right)_{1}-i\left(p_{i}\right)_{2} \\
\left(p_{i}\right)_{1}+i\left(p_{i}\right)_{2} \\
1-\left(p_{i}\right)_{3}
\end{array}\right) .
\end{aligned}
$$

- Unitary dynamics $\rho_{f}=U \rho_{i} U^{\dagger}$ defines a trace-preserving positive map

$$
\begin{aligned}
A_{U}: & \rho_{i} \rightarrow \rho_{f}=A_{U}\left(\rho_{i}\right), \\
\left(\rho_{f}\right)_{r^{\prime} s^{\prime}}= & \sum_{r, s=1}^{n}\left(U \otimes U^{*}\right)_{r^{\prime} s^{\prime} ; r s}\left(\rho_{i}\right)_{r s}, \quad r^{\prime}, s^{\prime}=1,2, \ldots, n .
\end{aligned}
$$

- Matrix transposition given by

$$
A_{T}: \quad \rho_{i} \rightarrow \rho_{f}=A_{T}\left(\rho_{i}\right)=\rho_{i}^{T}
$$

is an example of trace-preserving positive map.

Given a positive map $A$ on $\mathcal{H}_{n}$ one may extend it to the map $A \otimes \mathbb{I}_{d}$ acting on the tensor product space $\mathcal{H}_{n} \otimes \mathcal{H}_{d}$ of a composite $n \times d$ system, where $\mathbb{I}_{d}$ denotes identity map on $\mathcal{H}_{d}$. Then the positive map $A$ acts only on the $n$-dimensional subsystem of the composite state. If the $A \otimes \mathbb{I}_{d}$ transforms a composite density matrix into a physical state for any $d$, then the map $A$ is said to be completely positive (CP). Otherwise, it is called not-completely positive (NCP). Thus, a positive map represents a bonafide state to state transformation if it is completely positive. 
Under the action of a map $A: \rho_{i} \Longrightarrow \rho_{f}=A\left(\rho_{i}\right)$, preservation of hermiticity i.e., $\left(\rho_{f}\right)_{s^{\prime} r^{\prime}}^{*}=\left(\rho_{f}\right)_{r^{\prime} s^{\prime}}$ and the unit trace condition $\sum_{r^{\prime}=1}^{n}\left(\rho_{f}\right)_{r^{\prime} r^{\prime}}=1$ result in the following constraints on the elements of the $n^{2} \times n^{2}$ process matrix $A$ :

$$
\begin{aligned}
& \left(\rho_{f}\right)_{r^{\prime} s^{\prime}}=\left(\rho_{f}\right)_{s^{\prime} r^{\prime}}^{*} \Longrightarrow \sum_{r, s=1}^{n} A_{s^{\prime} r^{\prime} ; s r}^{*}\left(\rho_{i}\right)_{s r}^{*}=\sum_{r, s=1}^{n} A_{r^{\prime} s^{\prime} ; r s}\left(\rho_{i}\right)_{r s} \\
& \Longrightarrow \quad \sum_{r, s=1}^{n} A_{s^{\prime} r^{\prime} ; s r}^{*}\left(\rho_{i}\right)_{r s}=\sum_{r, s=1}^{n} A_{r^{\prime} s^{\prime} ; r s}\left(\rho_{i}\right)_{r s} \\
& \Longrightarrow \quad A_{r^{\prime} s^{\prime} ; r s}^{*}=A_{s^{\prime} r^{\prime} ; s r}
\end{aligned}
$$

and

$$
\begin{gathered}
\sum_{r^{\prime}=1}^{n}\left(\rho_{f}\right)_{r^{\prime} r^{\prime}}=1 \Longrightarrow \sum_{r^{\prime}, r, s} A_{r^{\prime} r^{\prime} ; r s}^{*}\left(\rho_{i}\right)_{r s} \\
\Longrightarrow \quad \sum_{r^{\prime}=1}^{n} A_{r^{\prime} r^{\prime} ; r s}=\delta_{r s}
\end{gathered}
$$

where $\delta_{r s}$ denotes Kronecker delta symbol.

A realigned process matrix $B$ was defined as [1,2]

$$
B_{r^{\prime} r ; s^{\prime} s}=A_{r^{\prime} s^{\prime} ; r s} .
$$

so that the hermiticity and unit trace conditions $(8),(9)$ on the $A$-form can be expressed as

$$
\begin{aligned}
B_{r^{\prime} r ; s^{\prime} s} & =B_{s^{\prime} s ; r^{\prime} r}^{*} \\
\sum_{r^{\prime}=1}^{n} B_{r^{\prime} r ; r^{\prime} s}=\delta_{r s} & \Longrightarrow \operatorname{Tr} B=n .
\end{aligned}
$$

Thus, a physically valid $A$-form requires that the corresponding realigned matrix $B$ (given by $(10 p)$ is a $n^{2} \times n^{2}$ Hermitian matrix with trace $n$.

Furthermore, positivity of the density matrix $\rho_{f}=$ $A\left(\rho_{i}\right) \geq 0$ leads to the following constraints on the elements of $A$ and $B$ respectively [1]:

$$
\begin{aligned}
& \sum_{r, s, r^{\prime}, s^{\prime}} x_{r}^{*} x_{s} A_{r s ; r^{\prime} s^{\prime}} y_{r^{\prime}} y_{s^{\prime}}^{*} \geq 0, \\
& \sum_{r, s, r^{\prime}, s^{\prime}} x_{r}^{*} y_{r^{\prime}} B_{r r^{\prime} ; s s^{\prime}} x_{s} y_{s^{\prime}}^{*} \geq 0 .
\end{aligned}
$$

In other words, positivity $\rho_{f}=A\left(\rho_{i}\right) \geq 0$ of the density matrix requires that $B \geq 0$.

It is pertinent to point out that the $B$-form is represented by a Hermitian matrix whereas $A$ is not; positivity of the $B$ matrix highlights that the output density matrix is legitimate. For this reason Sudarshan, Mathews and Rau [1] highlighted that the matrix B incorporates the kinematical restrictions on the dynamical law in a succinct fashion; we shall call $B$ the dynamical matrix. The $A$-form was used in Ref. [1] to define a linear map from input to output density operators (where the elements of the input and outpur density matrices are arranged in the form of $n^{2}$ component columns). Beyond this initial definition, the $A$ matrix was not recognized to have any clear role. Our focus here is to unravel the $A$-form to its full potential. We show in the next section that the $A$ matrix introduced in the Sudarshan-Mathew-Rau paper exhibits an elegant canonical structure and it reveals itself as a powerful tool in capturing all the dynamical features reflected by the corresponding $B$-form [7].

\section{Canonical structure of the A-form}

Consider an orthonormal set

$$
\left\{T_{\mu}, \mu=0,1,2, \ldots, n^{2}-1\right\}
$$

of $n \times n$ matrices satisfying

$$
\operatorname{Tr}\left[T_{\mu}^{\dagger} T_{\nu}\right]=\delta_{\mu, \nu} .
$$

We then construct a basis set

$$
\left\{T_{\mu} \otimes T_{\nu}^{*}, \mu, v=0,1, \ldots, n^{2}-1\right\}
$$

of $n^{2} \times n^{2}$ matrices and express the $A$ matrix (see (3)) as follows:

$$
A=\sum_{\mu, v=0}^{n^{2}-1} a_{\mu \nu} T_{\mu} \otimes T_{v}^{*}
$$

where the expansion coefficients $a_{\mu \nu}$ are given by

$$
a_{\mu v}=\operatorname{Tr}\left[A\left(T_{\mu}^{\dagger} \otimes T_{v}^{T}\right)\right], \quad \mu, v=0,1, \ldots, n^{2}-1 .
$$

The matrix elements $A_{r^{\prime} s^{\prime} ; r s}$ of the $A$ matrix are then given by

$$
A_{r^{\prime} s^{\prime} ; r s}=\sum_{\mu, v=0}^{n^{2}} a_{\mu \nu}\left[T_{\mu}\right]_{r^{\prime} r}\left[T_{\nu}^{*}\right]_{s^{\prime} s} .
$$

Let us examine the hermiticity preserving condition (8) on the expansion coefficients $a_{\alpha \beta}$ :

$$
A_{r^{\prime} s^{\prime} ; r s}^{*}=A_{s^{\prime} r^{\prime} ; s r} \Longrightarrow a_{\mu \nu}^{*}=a_{\nu \mu}
$$


In other words, the coefficients $a_{\mu \nu}, \mu, v=0,1, \ldots, n^{2}-1$ constitute a $n^{2} \times n^{2}$ Hermitian matrix, which we denote by $\mathcal{A}$.

Let $\mathcal{U}$ be a unitary matrix which diagonalizes $\mathcal{A}$, i.e.,

$$
\mathcal{U} \mathcal{A} \mathcal{U}^{\dagger}=\mathcal{A}_{0}=\left(\begin{array}{lllll}
\lambda_{0} & 0 & \ldots & \ldots & 0 \\
0 & \lambda_{1} & \ldots & \ldots & 0 \\
\vdots & \vdots & \ddots & & \vdots \\
0 & 0 & \ldots & \ldots & \lambda_{n^{2}-1}
\end{array}\right)
$$

where $\lambda_{\mu}, 0 \leq \mu \leq n^{2}-1$ denote the eigenvalues of $\mathcal{A}$. Thus we obtain

$$
\begin{aligned}
a_{\mu v} & =\left(\mathcal{U}^{\dagger} \mathcal{A}_{0} \mathcal{U}\right)_{\mu v} \\
& =\sum_{\alpha} \lambda_{\alpha} u_{\alpha \mu}^{*} u_{\alpha v}
\end{aligned}
$$

Substituting (21) in (16) we obtain the following canonical structure of the $A$ matrix:

$$
\begin{aligned}
A & =\sum_{\mu, v, \alpha} \lambda_{\alpha} u_{\alpha \mu}^{*} u_{\alpha \nu}\left(T_{\mu} \otimes T_{v}^{*}\right) \\
& =\sum_{\alpha} \lambda_{\alpha}\left(C_{\alpha} \otimes C_{\alpha}^{*}\right)
\end{aligned}
$$

where we have denoted

$$
C_{\alpha}=\sum_{\mu=0}^{n^{2}-1} u_{\alpha \mu}^{*} T_{\mu}
$$

- Using (22) we can express the matrix elements of $A$ as

$$
A_{r^{\prime} s^{\prime} ; r s}=\sum_{\alpha=0}^{n^{2}-1} \lambda_{\alpha}\left(C_{\alpha}\right)_{r^{\prime} r}\left(C_{\alpha}^{*}\right)_{s^{\prime} s} .
$$

Substituting (24) in (3) and simplifying, we obtain the following elegant structure for the action of the linear $A$-map on the column vector consisting of the elements of the input density matrix $\rho_{i}$ :

$$
\begin{aligned}
\left(\rho_{f}\right)_{r^{\prime} s^{\prime}} & =\sum_{r, s} A_{r^{\prime} s^{\prime} ; r s}\left(\rho_{i}\right)_{r s} \\
& =\sum_{r, s, \alpha} \lambda_{\alpha}\left(C_{\alpha}\right)_{r^{\prime} r}\left(C_{\alpha}^{*}\right)_{s^{\prime} s}\left(\rho_{i}\right)_{r s} \\
& =\sum_{r, s, \alpha} \lambda_{\alpha}\left(C_{\alpha}\right)_{r^{\prime} r}\left(\rho_{i}\right)_{r s}\left(C_{\alpha}^{\dagger}\right)_{s s^{\prime}} \\
& =\sum_{\alpha} \lambda_{\alpha}\left(C_{\alpha} \rho_{i} C_{\alpha}^{\dagger}\right)_{r^{\prime} s^{\prime}} \\
\Longrightarrow \rho_{f} & =\sum_{\mu} \lambda_{\mu} C_{\mu} \rho_{i} C_{\mu}^{\dagger} .
\end{aligned}
$$

- From (25) the trace preservation condition (9) assumes the form

$$
\begin{aligned}
& \operatorname{Tr}\left(\rho_{f}\right)=1 \\
& \Longrightarrow \sum_{\alpha} \lambda_{\alpha} \operatorname{Tr}\left(C_{\alpha} \rho_{i} C_{\alpha}^{\dagger}\right)=\sum_{\alpha} \lambda_{\alpha} \operatorname{Tr}\left(C_{\alpha}^{\dagger} C_{\alpha} \rho_{i}\right)=1 \\
& \Longrightarrow \sum_{\alpha} \lambda_{\alpha} C_{\alpha}^{\dagger} C_{\alpha}=I_{n} .
\end{aligned}
$$

- From (10) and (24) we may identify the elements of the realigned $B$ matrix as

$$
B_{r^{\prime} r ; s^{\prime} s}=\sum_{\alpha} \lambda_{\alpha}\left(C_{\alpha}\right)_{r^{\prime} r}\left(C_{\alpha}^{*}\right)_{s^{\prime} s}
$$

which happens to be the spectral decomposition of the dynamical $B$ matrix with $\lambda_{\mu}$ being its eigenvalues.

Highlighting point here is that 27) brings out an explicit connection between the Hermitian (coefficient) matrix $\mathcal{A}$ (see (16) and (19p) and the dynamical matrix $B$ of Ref. [1]:

1. The eigenvalues of the coefficient matrix $\mathcal{A}$ associated with the $A$-form are identically same as those of $B$.

2. A completely positive map requires that the coefficient matrix $\mathcal{A}$ is positive (i.e., the eigenvalues $\lambda_{\alpha}$ are non-negative whenever the map is completely positive).

3. In the case of a completely positive map one may define a set $\left\{E_{\alpha}, \alpha=0,1, \ldots, n^{2}-1\right\}$ of $n \times n$ matrices based on the canonical structure (22) of the $A$-map:

$$
E_{\alpha}=\sqrt{\lambda_{\alpha}} C_{\alpha} .
$$

Then the transformation $\rho_{i} \rightarrow \rho_{f}=A\left(\rho_{i}\right)$ gets expressed in terms of the Kraus operator-sum representation [8], i.e.,

$$
\begin{aligned}
\left(\rho_{f}\right)_{r^{\prime} s^{\prime}}= & \sum_{r, s} A_{r^{\prime} s^{\prime} ; r s}\left(\rho_{i}\right)_{r s} \\
= & \sum_{r, s, \alpha} \lambda_{\alpha}\left(E_{\alpha}\right)_{r^{\prime} r}\left(E_{\alpha}^{*}\right)_{s^{\prime} s}\left(\rho_{i}\right)_{r s} \\
= & \sum_{\alpha}\left(E_{\alpha}\right)_{r^{\prime} r}\left(\rho_{i}\right)_{r s}\left(E_{\alpha}^{\dagger}\right)_{s s^{\prime}} \\
= & \sum_{\alpha}\left(E_{\alpha} \rho_{i} E_{\alpha}^{\dagger}\right)_{r^{\prime} s^{\prime}} \\
& \Longrightarrow \rho_{f}=\sum_{\alpha} E_{\alpha} \rho_{i} E_{\alpha}^{\dagger} .
\end{aligned}
$$

We point out that the operator sum representation (29) was already described (via the spectral decomposition of the dynamical matrix $B$ ) by Sudarshan, 
Mathews and Rau in their 1961 paper [1] and it was independently proposed by Kraus [8] after 10 years. The operators $E_{\alpha}$ (see $28,,(29)$ ) associated with a completely positive map are known as Kraus operators in the literature.

Summarizing, in this section we have shown that the canonical structure 22 of the $A$-form plays a significant role on its own - bringing forth all the required features of the quantum channel-without any necessity to invoke the realigned $B$-form. In the next section we employ the $A$-form to elucidate the completely positive or not completely positive behavior of some familiar qubit channels.

\section{Canonical A-form of standard qubit maps}

In this section we illustrate explicit $4 \times 4$ matrix forms of the canonical $A$-form and its equivalence with the dynamical matrix $B$ of some standard qubit transformations.

\subsection{Unitary map}

Under the action of a unitary transformation we have

$$
\rho_{f}=U \rho_{i} U^{\dagger} \Longrightarrow\left(\rho_{f}\right)_{r^{\prime} s^{\prime}}=\sum_{r, s} U_{r^{\prime} r} U_{s^{\prime} s}^{*}\left(\rho_{i}\right)_{r s}=\sum_{r, s}\left(U \otimes U^{*}\right)_{r^{\prime} s^{\prime} ; r s}\left(\rho_{i}\right)_{r s}
$$

We thus obtain (see (3))

$$
\left(A_{U}\right)_{r^{\prime} s^{\prime} ; r s}=\left(U \otimes U^{*}\right)_{r^{\prime} s^{\prime} ; r s} \Longrightarrow A_{U}=U \otimes U^{*}
$$

Let us consider the $2 \times 2$ unitary matrix

$$
\begin{aligned}
U & =e^{i(\vec{\sigma} \cdot \hat{n}) \theta / 2},|\hat{n}|^{2}=n_{1}^{2}+n_{2}^{2}+n_{3}^{2}=1 \\
& =I_{2} \cos (\theta / 2)+i \vec{\sigma} \cdot \hat{n} \sin (\theta / 2) \\
& =\left(\begin{array}{cc}
\cos \left(\frac{\theta}{2}\right)+i n_{3} \sin \left(\frac{\theta}{2}\right) & i n_{-} \sin \left(\frac{\theta}{2}\right) \\
i n_{+} \sin \left(\frac{\theta}{2}\right) & \cos \left(\frac{\theta}{2}\right)-i n_{3} \sin \left(\frac{\theta}{2}\right)
\end{array}\right), n_{ \pm}=\left(n_{1} \pm i n_{2}\right) .
\end{aligned}
$$

Then the matrix $A_{U}$ (see $(6,231)$ associated with the unitary matrix (32) takes the form

$$
A_{U}=\left(I_{2} \otimes I_{2}\right) \cos ^{2}(\theta / 2)+\left(\vec{\sigma} \cdot \hat{n} \otimes \vec{\sigma}^{*} \cdot \hat{n}\right) \sin ^{2}(\theta / 2)-i\left(I_{2} \otimes \vec{\sigma}^{*} \cdot \hat{n}-\vec{\sigma} \cdot \hat{n} \otimes I_{2}\right) \cos (\theta / 2) \sin (\theta / 2) .
$$

Denoting $\sigma_{0}=I_{2}$ and employing the basis set $\left\{T_{\mu}=\frac{1}{\sqrt{2}} \sigma_{\mu}\right\}$ of $2 \times 2$ matrices satisfying (see $(14)$ ) the conditions

$$
\frac{1}{2} \operatorname{Tr}\left[\sigma_{\mu} \sigma_{v}\right]=\delta_{\mu \nu}, \mu, v=0,1,2,3,
$$

we express 33 in the following compact form.

$$
A_{U}=\frac{1}{2} \sum_{\mu, v=0,1,2,3}\left(a_{U}\right)_{\mu \nu} \sigma_{\mu} \otimes \sigma_{\nu}^{*},
$$

Observe that

$$
\begin{aligned}
\left(a_{U}\right)_{\mu \nu} & =\frac{1}{2} \operatorname{Tr}\left[A_{U}\left(\sigma_{\mu} \otimes \sigma_{v}^{*}\right)\right] \\
& =\frac{1}{2} \operatorname{Tr}\left[U \sigma_{\mu}\right] \operatorname{Tr}\left[U \sigma_{\nu}\right]^{*}
\end{aligned}
$$

where $\operatorname{Tr}\left[U \sigma_{\mu}\right], \mu=0,1,2,3$ is evaluated using 32 :

$$
\begin{aligned}
& \operatorname{Tr}\left[U \sigma_{0}\right]=2 \cos \left(\frac{\theta}{2}\right), \\
& \operatorname{Tr}\left[U \sigma_{k}\right]=2 i n_{k} \sin \left(\frac{\theta}{2}\right), \quad k=1,2,3
\end{aligned}
$$

Then the $4 \times 4$ coefficient matrix $\mathcal{A}_{U}=\left(\left(a_{U}\right)_{\mu \nu}\right)($ see 35 $)$ associated with $A_{U}$ is given by

$$
\mathcal{A}_{U}=2 X_{U} X_{U}^{\dagger}, \quad X_{U}=\left(\begin{array}{c}
\cos \left(\frac{\theta}{2}\right) \\
i n_{1} \sin \left(\frac{\theta}{2}\right) \\
i n_{2} \sin \left(\frac{\theta}{2}\right) \\
i n_{3} \sin \left(\frac{\theta}{2}\right)
\end{array}\right)
$$

From (37) it is seen that the coefficient matrix $\mathcal{A}_{U}$ is a rank-1 positive matrix with eigenvalue 2 and eigenvector $X_{U}$. The realigned $B_{U}$ matrix matches exactly with the coefficient matrix $\mathcal{A}_{U}$, i.e.,

$$
B_{U}=2 X_{U} X_{U}^{\dagger} \equiv \mathcal{A}_{U}
$$




\subsection{Pin map}

Consider a linear $A$-form mapping every input state $\rho_{i}$ to a fixed output state $\rho_{0}$, i.e.,

$$
A_{\text {pin }}: \rho_{i} \rightarrow \rho_{0}=A_{\text {pin }}\left(\rho_{i}\right) \forall \rho_{i} .
$$

Sudarshan, Mathews and Rau presented this map in terms of the $B$-form, which was termed as relaxation generator.
Here we would like to illustrate the canonical structure of the $A$-form associated with the qubit pin-map.

Let the fixed output density matrix of the qubit be given by

$$
\rho_{0}=\frac{1}{2}\left(I_{2}+\vec{\sigma} \cdot \vec{p}_{0}\right)
$$

The $4 \times 4$ matrix $A_{\text {pin }}$ corresponding to the pin map is identified as follows:

$$
\begin{gathered}
\left(\begin{array}{c}
1+\left(p_{0}\right)_{3} \\
\left(p_{0}\right)_{1}-i\left(p_{0}\right)_{2} \\
\left(p_{0}\right)_{1}+i\left(p_{0}\right)_{2} \\
1-\left(p_{0}\right)_{3}
\end{array}\right)= \\
\quad\left(\begin{array}{llll}
\left(A_{\text {pin }}\right)_{00 ; 00} & \left(A_{\text {pin }}\right)_{00 ; 01} & \left(A_{\text {pin }}\right)_{00 ; 10} & \left(A_{\text {pin }}\right)_{00 ; 11} \\
\left(A_{\text {pin }}\right)_{01 ; 00} & \left(A_{\text {pin }}\right)_{01 ; 01} & \left(A_{\text {pin }}\right)_{01 ; 10} & A_{01 ; 11} \\
\left(A_{\text {pin }}\right)_{10 ; 00} & \left(A_{\text {pin }}\right)_{10 ; 01} & \left(A_{\text {pin }}\right)_{10 ; 10} & \left(A_{\text {pin }}\right)_{10 ; 11} \\
\left(A_{\text {pin }}\right)_{11 ; 00} & \left(A_{\text {pin }}\right)_{11 ; 01} & \left(A_{\text {pin }}\right)_{11 ; 10} & \left(A_{\text {pin }}\right)_{11 ; 11}
\end{array}\right)\left(\begin{array}{c}
1+\left(p_{i}\right)_{3} \\
\left(p_{i}\right)_{1}-i\left(p_{i}\right)_{2} \\
\left(p_{i}\right)_{1}+i\left(p_{i}\right)_{2} \\
1-\left(p_{i}\right)_{3}
\end{array}\right) \forall \vec{p}_{i} \\
\Longrightarrow A_{\text {pin }}=\frac{1}{2}\left(\begin{array}{cccc}
1+\left(p_{0}\right)_{3} & 0 & 0 & 1+\left(p_{0}\right)_{3} \\
\left(p_{0}\right)_{1}-i\left(p_{0}\right)_{2} & 0 & 0 & \left(p_{0}\right)_{1}-i\left(p_{0}\right)_{2} \\
\left(p_{0}\right)_{1}+i\left(p_{0}\right)_{2} & 0 & 0 & \left(p_{0}\right)_{1}+i\left(p_{0}\right)_{2} \\
1-\left(p_{0}\right)_{3} & 0 & 0 & 1-\left(p_{0}\right)_{3}
\end{array}\right)
\end{gathered}
$$

Using the orthonormal basis set of matrices $\left\{\frac{\sigma_{\mu}}{\sqrt{2}}, \mu=0,1,2,3\right\}$ we expand

$$
A_{\text {pin }}=\frac{1}{2} \sum_{\mu, v}\left(a_{\text {pin }}\right)_{\mu \nu} \sigma_{\mu} \otimes \sigma_{v}^{*}, \text { where }\left(a_{\text {pin }}\right)_{\mu \nu}=\frac{1}{2} \operatorname{Tr}\left[A_{\text {pin }}\left(\sigma_{\mu} \otimes \sigma_{v}^{*}\right)\right] .
$$

The coefficient matrix $\mathcal{A}_{\text {pin }}=\left(\left(a_{\text {pin }}\right)_{\mu \nu}\right)$ is then found to be

$$
\mathcal{A}_{\text {pin }}=\frac{1}{2}\left(\begin{array}{cccc}
1 & \left(p_{0}\right)_{1} & \left(p_{0}\right)_{2} & \left(p_{0}\right)_{3} \\
\left(p_{0}\right)_{1} & 1 & -i\left(p_{0}\right)_{3} & i\left(p_{0}\right)_{2} \\
\left(p_{0}\right)_{2} & i\left(p_{0}\right)_{3} & 1 & -i\left(p_{0}\right)_{1} \\
\left(p_{0}\right)_{3} & -i\left(p_{0}\right)_{2} & i\left(p_{0}\right)_{1} & 1
\end{array}\right) .
$$

The eigenvalues of $\mathcal{A}_{\text {pin }}$ are given by

$$
\lambda_{0}=\lambda_{1}=\frac{1}{2}\left(1+\left|\vec{p}_{0}\right|\right), \quad \lambda_{2}=\lambda_{3}=\frac{1}{2}\left(1-\left|\vec{p}_{0}\right|\right) .
$$

Clearly, the eigenvalues of $\mathcal{A}_{\text {pin }}$ are all positive ensuring that the pin map is completely positive.

The $B$ matrix associated with the pin map is constructed using the explicit matrix form of $A_{\text {pin }}$ (see (41)):

$$
\begin{aligned}
B_{\text {pin }} & =\frac{1}{2}\left(\begin{array}{cccc}
1+\left(p_{0}\right)_{3} & 0 & \left(p_{0}\right)_{1}-i\left(p_{0}\right)_{2} & 0 \\
0 & 1+\left(p_{0}\right)_{3} & 0 & \left(p_{0}\right)_{1}-i\left(p_{0}\right)_{2} \\
\left(p_{0}\right)_{1}+i\left(p_{0}\right)_{2} & 0 & 1-\left(p_{0}\right)_{3} & 0 \\
0 & \left(p_{0}\right)_{1}+i\left(p_{0}\right)_{2} & 0 & 1-\left(p_{0}\right)_{3}
\end{array}\right) \\
& =\rho_{0} \otimes I_{2}
\end{aligned}
$$

Eigenvalues of $B_{\text {pin }}$ match with those of the coefficient matrix $\mathcal{A}_{\text {pin }}$ (see $(43)$ ), thus establishing the equivalence between the two. 


\subsection{Transpose map}

Consider the transpose map $A_{T}: \rho \rightarrow \rho^{T}$ on qubit density matrices (see (4)). We obtain the associated $4 \times 4$ matrix form of $A_{T}$ as

$$
A_{T}=\left(\begin{array}{cccc}
1 & 0 & 0 & 0 \\
0 & 0 & 1 & 0 \\
0 & 1 & 0 & 0 \\
0 & 0 & 0 & 1
\end{array}\right)
$$

Employing the basis set $\left\{\frac{\sigma_{\mu}}{\sqrt{2}}, \mu=0,1,2,3\right\}$ we express

$$
A_{T}=\frac{1}{2} \sum_{\mu, \nu=0}^{3}\left(a_{T}\right)_{\mu \nu}\left(\sigma_{\mu} \otimes \sigma_{\nu}^{*}\right)
$$

to obtain the following explicit structure for the coefficient matrix $\mathcal{A}_{T}$ :

$$
\mathcal{A}_{T}=\operatorname{diag}(-1,1,1,1) .
$$

The matrix $\mathcal{A}_{T}$ is not positive (one of the eigenvalues of $\mathcal{A}_{T}$ is -1) which points towards the not-completely positive nature of the transpose map.

From the explicit matrix structure of $A_{T}$ (see (45p) it is easy to see that the realigned dynamical matrix $B_{T} \equiv A_{T}$. The eigenvalues of $B_{T}$ match with those of the coefficent matrix $\mathcal{A}_{T}$ (see (47)).

\subsection{Projection of the Bloch sphere onto its equatorial plane}

A map that projects the entire Bloch sphere onto the equatorial plane is defined by the transformation of the Bloch vector

$$
\left(p_{1}, p_{2}, p_{3}\right) \rightarrow\left(p_{1}, p_{2}, 0\right) .
$$

This leads to the following linear transformation

$$
\left(\begin{array}{c}
1 \\
p_{1}-i p_{2} \\
p_{1}+i p_{2} \\
1
\end{array}\right)=\frac{1}{2}\left(\begin{array}{llll}
1 & 0 & 0 & 1 \\
0 & 2 & 0 & 0 \\
0 & 0 & 2 & 0 \\
1 & 0 & 0 & 1
\end{array}\right)\left(\begin{array}{c}
1+p_{3} \\
p_{1}-i p_{2} \\
p_{1}+i p_{2} \\
1-p_{3}
\end{array}\right)
$$

We then express $A_{P}=\frac{1}{2} \sum_{\mu, \nu}\left(a_{P}\right)_{\mu \nu}\left(\sigma_{\mu} \otimes \sigma_{v}^{*}\right)$ to obtain

$$
\mathcal{A}_{P}=\frac{1}{2} \operatorname{diag}(3,1,1,-1) .
$$

Negative eigenvalues of $\mathcal{A}_{P}$ clearly indicate that projection of the Bloch sphere onto the equatorial plane is not physical as it corresponds to a not completely positive map. The dynamical matrix $B_{P}$ is then obtained using the realignment $\left(B_{P}\right)_{r^{\prime} r ; s^{\prime} s}=\left(A_{P}\right)_{r^{\prime} s^{\prime} ; r s}$ :

$$
B_{P}=\frac{1}{2}\left(\begin{array}{cccc}
1 & 0 & 0 & 2 \\
0 & 1 & 0 & 0 \\
0 & 0 & 1 & 0 \\
2 & 0 & 0 & 1
\end{array}\right)
$$

Eigenvalues of $B_{P}$ are same as those of $\mathcal{A}_{P}$.

\subsection{Bit flip channel}

A qubit bit flip channel reverses the state of a qubit from $|0\rangle$ to $|1\rangle$ with probability $1-p, 0 \leq p \leq 1$; the channel keeps the states unaltered with a probability $p$. This is a completely positive map equipped with the Kraus operators given by [3]

$$
E_{0}=\sqrt{p}\left(\begin{array}{cc}
1 & 0 \\
0 & 1
\end{array}\right), \quad E_{1} \equiv \sqrt{1-p}\left(\begin{array}{cc}
0 & 1 \\
1 & 0
\end{array}\right) .
$$

Using the operator-sum representation $\rho_{f}=E_{0} \rho_{i} E_{0}^{\dagger}+$ $E_{1} \rho_{i} E_{1}^{\dagger}$ we construct the associated $A$ matrix:

$$
A_{\mathrm{BF}}=\left(\begin{array}{cccc}
p & 0 & 0 & 1-p \\
0 & p & 1-p & 0 \\
0 & 1-p & p & 0 \\
1-p & 0 & 0 & p
\end{array}\right)
$$

Adopting the matrix basis $\left\{\frac{\sigma_{\mu}}{\sqrt{2}}, \mu=0,1,2,3\right\}$, as in all other examples studied earlier, we compute the coefficient matrix $\mathcal{A}_{\mathrm{BF}}=\left(a_{\mu \nu}\right)$ associated with $A_{\mathrm{BF}}$ :

$$
\mathcal{A}_{\mathrm{BF}}=\left(\begin{array}{cccc}
2 p & 0 & 0 & 0 \\
0 & 2(1-p) & 0 & 0 \\
0 & 0 & 0 & 0 \\
0 & 0 & 0 & 0
\end{array}\right)
$$

Note that the eigenvalues $2 p, 2(1-p)$ of $\mathcal{A}_{\mathrm{BF}}$ are positive and ascertain the completely positive nature of the bit flip channel.

\subsection{Phase flip channel}

A qubit phase flip channel is equipped with the Kraus operators [3]

$$
E_{0}=\sqrt{p}\left(\begin{array}{ll}
1 & 0 \\
0 & 1
\end{array}\right), \quad E_{1}=\sqrt{1-p}\left(\begin{array}{cc}
1 & 0 \\
0 & -1
\end{array}\right) .
$$

The linear map $A_{\mathrm{PF}}$ associated with the phase flip channel is given explicitly as a $4 \times 4$ matrix form:

$$
A_{\mathrm{PF}}=\frac{1}{2}\left(\begin{array}{cccc}
1 & 0 & 0 & 0 \\
0 & 2 p-1 & 0 & 0 \\
0 & 0 & 2 p-1 & 0 \\
0 & 0 & 0 & 1
\end{array}\right) .
$$


Then the associated coefficent matrix $\mathcal{A}_{\mathrm{PF}}$ is found to be

$$
\mathcal{A}_{\mathrm{PF}}=\left(\begin{array}{cccc}
2 p & 0 & 0 & 0 \\
0 & 0 & 0 & 0 \\
0 & 0 & 0 & 0 \\
0 & 0 & 0 & 2(1-p)
\end{array}\right)
$$

The eigenvalues $2 p, 2(1-p)$ of $\mathcal{A}_{\mathrm{PF}}$ are necessarily positive and confirm the legitimacy (complete positivity) of the phase flip channel.

\section{Summary}

The dynamical $A$ and $B$-maps were pioneered by Sudarshan, Mathews and Rau [1] in the context of open system dynamics. Unfortunately this seminal 1961 paper did not receive its due attention in the field, although it contained all the details of finite dimensional quantum channels. In this work we have elaborated on the canonical structure of the $A$-form, establishing that it offers an alternative approach to recognize the completely positive/not completely positive nature of quantum channels. We have illustrated the canonical structure of the $A$ map in several standard examples of qubit maps. A new geometrical representation based on the Lorentz singular value decomposition [9] of the canonical $A$-form associated with qubit transformations is being prepared and it will be presented separately as a sequel to the present work.

\section{Dedication}

We dedicate this tutorial article as a mark of our reverence to Professor E. C. G. Sudarshan.

\section{Acknowledgement}

A. R. Usha Devi, B. N. Karthik and Sudha acknowledge financial support from the Department of Science and Technology, India (Project No. DST/ICPS/QuST/Theme2/Q107/2019).

\section{References}

[1] E. C. G. Sudarshan, P. M. Mathews, J. Rau. Stochastic dynamics of quantum-mechanical systems. Physical Review 1961; 121(3):920-924. doi:10.1103/ PhysRev.121.920.

[2] T. F. Jordan, E. C. G. Sudarshan. Dynamical mappings of density operators in quantum mechanics. Journal of Mathematical Physics 1961; 2(6):772-775. doi:10.1063/1.1724221.

[3] M. A. Nielsen, I. L. Chuang. Quantum Computation and Quantum Information. Cambridge University Press, Cambridge, 2010. doi:10.1017/ cbo9780511976667.

[4] H.-P. Breuer, F. Petruccione. The Theory of Open Quantum Systems. Oxford University Press, Oxford, 2007. doi:10.1093/acprof:oso/ 9780199213900.001 .0001

[5] S. Simon, S. P. Rajagopalan, R. Simon. The structure of states and maps in quantum theory. Pramana 2009; 73(3):471. doi : 10.1007/s12043-009-0100-1.

[6] V. Jagadish, F. Petruccione. An invitation to quantum channels. Quanta 2018; 7:54-67. doi : 10.12743/ quanta.v7i1.77.

[7] A. R. Usha Devi, A. K. Rajagopal, Sudha. Open-system quantum dynamics with correlated initial states, not completely positive maps, and non-Markovianity. Physical Review A 2011; 83(2):022109. doi: 10.1103/PhysRevA.83.022109.

[8] K. Kraus. General state changes in quantum theory. Annals of Physics 1971; 64(2):311-335. doi:10. 1016/0003-4916(71)90108-4.

[9] Sudha, H. S. Karthik, R. Pal, K. S. Akhilesh, S. Ghosh, K. S. Mallesh, A. R. Usha Devi. Canonical forms of two-qubit states under local operations. Physical Review A 2020; 102(5):052419. doi: 10.1103/PhysRevA.102.052419. 